Originally published as:

Hellenbrand, W., Elias, J., Wichmann, O., Dehnert, M., Frosch, M., Vogel, U.

Epidemiology of invasive meningococcal disease in Germany, 2002-2010, and impact of vaccination with meningococcal $\mathrm{C}$ conjugate vaccine

(2013) Journal of Infection, 66 (1), pp. 48-56.

AUTHOR MANUSCRIPT. () Elsevier (2012): This is the author's version of the work. It is posted here by permission of Elsevier for personal use, not for redistribution. Some changes resulting from the publishing process, such as editing, corrections, structural formatting, and other quality control mechanisms may not be reflected in this document. Some changes may have been made to this work since being accepted for publication. A definitive version was subsequently published in Journal of Infection, [Volume: 66, Issue: 1, 2012 Oct 5] DOI: 10.1016/j.jinf.2012.09.008 


\title{
Epidemiology of invasive meningococcal disease in Germany, 2002-2010, and impact of vaccination with meningococcal $C$ conjugate vaccine
}

\author{
Wiebke Hellenbrand $^{\mathrm{a}}$, Johannes Elias ${ }^{\mathrm{b}}$, Ole Wichmann ${ }^{\mathrm{a}}$, Manuel Dehnert ${ }^{\mathrm{c}}$, Matthias Frosch ${ }^{\mathrm{b}}$, Ulrich \\ Vogel $^{b}$ \\ ${ }^{a}$ Immunization Unit, Department of Infectious Disease Epidemiology, Robert Koch Institute, \\ DGZ-Ring 1, 13086 Berlin, Germany \\ ${ }^{\mathrm{b}}$ University of Würzburg, Institute for Hygiene and Microbiology and National Reference Laboratory for \\ Meningococci, Josef-Schneider-Str. 2 (E1), Würzburg, Germany \\ ${ }^{c}$ Department of Infectious Disease Epidemiology, Robert Koch Institute, DGZ-Ring 1, 13086 Berlin, \\ Germany
}

\section{Summary}

Objectives: To analyse serogroup (Sg)- and finetype-specific invasive meningococcal disease burden (IMD) in Germany, 2002-2010, with emphasis on effects of vaccination with conjugate $\mathrm{SgC}$ vaccines targeting one-year old children since 2006, including individual-based catch-up to 17 years of age.

Methods: Serogroup- and age-specific IMD incidence and trends were calculated using statutory surveillance data. The national reference laboratory performed genetic finetyping. Vaccination uptake data were obtained from school entry surveys and prescription monitoring.

Results: In persons $<25$ years, $\mathrm{SgB}$ and $\mathrm{SgC}$ IMD incidence decreased significantly from 0.63 to $0.32 / 100,000$ and 0.26 to $0.10 / 100,000$, respectively. The decline was significantly steeper for $\mathrm{SgC}$ than $\mathrm{SgB}$ in 1-5 year-olds, the primary vaccination target group, but not other ages. The slope of the $\mathrm{SgC}$ incidence curves was similar before and after vaccination implementation in all age groups; however, the decrease in incidence was steeper in states with higher vaccination uptake. Declining $\mathrm{SgC}$ incidence was associated with decreased $\mathrm{SgC}$ finetype diversity. An increase in $\mathrm{SgY}$ incidence was limited to adults.

Conclusions: Results suggest effects of the German $\mathrm{SgC}$ vaccination strategy are limited, although interpretation is complicated by already low and decreasing incidence before vaccination. More effective use of vaccination resources might be achieved by rigorously targeting adolescents in addition to 1 -year-olds. 


\section{Introduction}

While the overall incidence of invasive meningococcal disease (IMD), caused by Neisseria meningitidis, is relatively low in Europe (0.1-3.4 cases/100,000 inhabitants), case-fatality remains high at $7-9 \% .{ }^{1}$ and 2 Compared to other European countries, incidence in Germany (81.8 million inhabitants) is low. ${ }^{1 \text { and } 3}$ Surveillance is based on statutory notification according to the German Protection Against Infection Act (IfSG) since 2001, with linkage to typing performed at the National Reference Laboratory for Meningococci (NRLM).

In 2006, the German Standing Committee on Vaccination (STIKO) recommended routine meningococcal serogroup $\mathrm{C}(\mathrm{SgC})$ vaccination in the second year of life. Previously, $\mathrm{SgC}$ conjugate vaccines were recommended only for risk groups, except in the federal state of Saxony (4.1 million inhabitants), where $\mathrm{SgC}$ vaccination was recommended from 2 months of age in July $2003 .{ }^{4}$ STIKO recommended catch-up vaccination for all children and adolescents $<18$ years on an individual basis, ${ }^{5}$ and 6 but a catch-up campaign was not undertaken.

Here, we analyse IMD burden and secular trends in Germany from 2002 to 2010, including analysis of vaccination effects.

\section{Materials and methods}

\section{SgC vaccination coverage}

Vaccination coverage with conjugate $\mathrm{SgC}$ vaccines is ascertained routinely by local health authorities (LHA) from children at school entry and provided to the Robert Koch Institute (RKI) annually in aggregate form. Furthermore, the number of prescribed conjugate $\mathrm{SgC}$ vaccine doses from 2006 to 2010 was analysed based on data purchased by RKI from Insight Health (http://www.insighthealth.de/), which collects data on all prescriptions for $>99 \%$ of persons insured by the statutory health insurance (SHI) in Germany from pharmacy data processing centres. SHI covers $~ 85 \%$ of the German population. ${ }^{7}$ We calculated the number of prescribed vaccine doses per 1000 one-year-old children, as these comprised the primary vaccination target group, for each state. We used the median value to categorize states as having high or low vaccination uptake.

\section{Surveillance}

According to IfSG, notification of IMD to LHA is required of physicians and laboratories. LHA verify notified cases according to the national case definition. Clinical criteria include at least one of fever, meningeal signs, skin lesions (maculopapular rash, petechiae, or ecchymoses), signs of increased intracranial pressure or circulatory collapse. Laboratory confirmation requires detection of $N$. meningitidis in blood, cerebrospinal fluid (CSF), skin biopsy of hemorrhagic infiltrates, or clinical specimens from a normally sterile site by culture, nucleic acid detection, microscopic detection of gram-negative diplococci or detection of capsular antigen (CSF only). Cases fulfilling clinical and either laboratory or epidemiological criteria (contact to laboratory-confirmed case) are considered confirmed, and transmitted from LHA to RKI via the state level in anonymized form together with data on vaccination status, clinical presentation (meningitis, sepsis, Waterhouse-Friderichsen Syndrome (WHF)), hospitalization, and outcome (survived/died).

\section{Laboratory methods}

NRLM routinely receives isolates or samples from IMD patients from peripheral laboratories and performs antigen sequence typing. ${ }^{8} \mathrm{~A}$ finetype is defined as the combination of serogroup, antigen sequence type of two variable regions (VR) of the outer membrane protein PorA and of one VR of FetA, resulting in the formula "Sg:PorA VR1,VR2:FetA VR". ${ }^{9}$ Since 2004, all NRLM results are reported directly to LHA in addition to primary laboratories. NRLM cases were matched to notified 
cases using an automated algorithm requiring identical sex, month/year of birth, county and state of residence, $\leq 7$ days between date of illness onset and date sample taken and a unique match. A manual search was performed for potential further matches fulfilling fewer criteria. These were verified at the level of the local health authority using personalized data not available at the national level.

\section{Data analysis}

IMD incidence was calculated using 2002-2010 notification data as of 4 July 2011 and population estimates from the Federal Statistical Office (http://www.destatis.de). Serogroup specific incidences were calculated assuming a similar serogroup distribution among cases with and without known serogroup. Proportions were compared using the Chi-squared test. Exact binomial confidence intervals were calculated for case-fatality estimates. Temporal trends were estimated using poisson and negative binomial regression analysis. Confidence bands $(\mathrm{Cl})$ were obtained using delta method.

Finetype diversity $D$ according to serogroup was calculated as

$D=\left(\sum_{j}^{s} n j(n j-1)\right) / N *(N-1)$

where $s=$ total number of finetypes, $n_{j}=$ number of strains belonging to the $j$ th finetype and $N=$ total number of cases in the serogroup under consideration. ${ }^{10}$ Confidence intervals $(\mathrm{Cl})$ were calculated according to Grundmann et al. ${ }^{11}$

Statistical analyses were performed using PASW SPSS 18 and STATA 11.0/IC.

\section{Results}

\section{$\mathrm{SgC}$ vaccination coverage}

Based on data from $92 \%$ of children entering school in 13 of 16 federal states (4-6-year-old children presenting with vaccination records, $n=494,455)$, $\mathrm{SgC}$ vaccination coverage in 2010 was $69,8 \%$ (range: $52.8 \%-89.5 \%$ ) (Table 1). In 2009, coverage was $55.9 \%$ (range in 13 states: $9.1 \%-79.5 \%$ ). ${ }^{12}$ These children would have been one year old in 2005-2007, making it likely that some were vaccinated at an older age. The number of annually prescribed $\mathrm{SgC}$ vaccine doses per 1000 oneyear-old children peaked in most states in 2007, the first full year after implementing the $\mathrm{SgC}$ vaccination recommendation in July 2006 (Table 1 ) with values $>1000 / 1000$ reflecting recommended catch-up vaccination in older children. We categorized vaccine uptake as low in the 8 states with rates below the median of $1858 / 1000$ and as high in the remaining states. The overall vaccination coverage at school entry in states classified as low was $61.1 \%$ and in states classified as high, $73.9 \%$.

\section{Description of surveillance data base}

From 2002 to 2010, 5073 confirmed IMD cases were reported to RKI. Of these, 3600 (71.0\%) were diagnosed by culture, $554(10.9 \%)$ by PCR, 745 (14.7\%) only by microscopy or antigen test, with no data for $114(2.2 \%)$. A total of 3653 NRLM cases were matched to notified cases, 3505 of which had complete results of genetic finetyping available. The percentage of NRLM cases without a match decreased from $11.5 \%$ in 2002 to $2.4 \%$ in 2006 and was $2.8 \%-3.3 \%$ in $2007-2010$. After matching to NRLM cases, the serogroup was available for $4488 / 5073$ notified cases $(88.5 \%$, increasing from $85.7 \%$ to $90.5 \%$ from 2002 to 2010 ). The notified and NRLM serogroup differed in 48 of the matched cases $(1.3 \%)$ and was corrected according to NRLM results. 


\section{Clinical presentation and case fatality}

The proportion of cases missing data on clinical presentation decreased from high levels shortly after IfSG-implementation (64.1\% in 2002; 55.2\% in 2003) to <10.5\% in 2004-2010. Therefore, description of clinical manifestations was limited to data from 2004 to $2010(n=3353)$. Meningitis was reported in $68.7 \%$ of cases and sepsis in $39.8 \%$ (both manifestations in $8.5 \%$ ). WHF was reported in $30.0 \%$ of cases with sepsis (12\% of all cases). Clinical presentation was similar in males and females, but adults $\geq 50$ years presented with sepsis more often $(51.5 \%)$ than younger persons, with the lowest frequency among $15-24$ year olds $(33.3 \%, p<0.0001)$. Compared to cases with $\mathrm{SgB}(38.2 \%)$, cases with SgC (45.4\%, $p=0.0007)$ and SgY (46.8\%, $p=0.07)$, but not SgW135 (36.1\%, $p=0.43)$, were more likely to present with sepsis. There was no difference in the percentage of cases presenting with WHF according to serogroup $(p=0.29)$.

Data on vital status was available for $99.3 \%$ of cases reported during 2002-2010. Of 441 reported deaths, $298(67.6 \%)$ occurred in persons <25 years. Case fatality (CF) was $8.8 \%(95 \% \mathrm{Cl}: 8.0-9.6 \%)$ overall, lowest in $5-14$ year olds $(4.2 \%)$ and highest in $\geq 50$-year olds (15.8\%). There was no difference in $\mathrm{CF}$ according to sex. CF was higher for $\mathrm{SgC}$ than $\mathrm{SgB}$ in all age groups (Table 2). A total of 15 and 13 deaths, respectively, occurred among cases with SgY (CF: $8.4 \%, 95 \% \mathrm{Cl}: 4.6-14.0 \%)$ and SgW135 (CF: $11.5 \%, 95 \% \mathrm{Cl}: 5.9-19.6 \%$ ).

\section{IMD disease burden according to age, serogroup, and region}

The mean annual IMD incidence from 2002 to 2010 was 0.71 cases $/ 100,000$ inhabitants. Annual incidence was highest in 2003 at $0.94 / 100,000$, decreasing to 0.47 in 2010. Mean IMD incidence was highest in infants $(11.93 / 100,000)$, declining to lower levels in children aged 5-14 (1.00/100,000). A smaller peak was seen in 15-19-year olds $(2.63 / 100,000)$, with a decrease to $0.82 / 100,000$ in $20-24$ year olds and lowest incidences in adults $>24$ years $(0.20 / 100,000)$. Mean IMD incidence was slightly higher in males than females $(0.75 / 100,000$ vs. $0.63 / 100,000, p<0.0001)$ except among $\geq 50$ yearolds, in whom the incidence in women $(0.23 / 100,000)$ was slightly higher than in men $(0.18 / 100,000$, $p<0.0001)$.

As shown in online Table 1, SgB occurred most frequently (67.9\%) followed by SgC (25.0\%), $\mathrm{SgY}$ (3.4\%) and SgW135 (2.2\%). SgB was most common among IMD cases in infants (80.1\%) and least common in children aged $5-14$ years $(60.9 \%)$. The proportion of cases with $\mathrm{SgC}$ was highest in children aged 5-14 years (32.8\%) and lowest in infants (16.7\%). The proportion of cases presenting with $\mathrm{SgY}$ and $\mathrm{SgW} 135$ was highest in adults $>50$ years $(10.8 \%$ and $6.2 \%$, respectively). There was a significant decrease in the proportion of SgC IMD from 2006 to 2010 overall (29.3\%-21.9\%, $\left.p_{\text {trend }}=0.02\right)$ and in 6-14 year-old children $\left(35.8 \%-15.8 \%, p_{\text {trend }}=0.02\right)$. The decrease in the proportion of SgC IMD in $1-5$ year olds $\left(27.4 \%-20.0 \%, p_{\text {trend }}=0.09\right)$ and $15-24$ year olds $(31.3 \%-$ $\left.21.3 \%, p_{\text {trend }}=0.08\right)$ did not reach significance, and there was no change in adults and infants.

The distribution of serogroups according to age (Figure 1) is relevant for assessment of potential vaccination impact. Thus, $17.1 \%$ of all $\mathrm{SgB}$ cases and $9.8 \%$ of $\mathrm{SgC}$ cases occurred in infants (which comprise $0.8 \%$ of the German population). The highest proportion of $\mathrm{SgB}$ and $\mathrm{SgC}$ cases occurred in $0-4$ and $15-19$ year-olds, and the highest proportion of SgY and W135 cases in adults $\geq 50$ years (Figure 1).

The decrease in total IMD incidence was reflected in significantly declining trends for $\mathrm{SgB}$ and $\mathrm{SgC}$ (Figure 2A). SgY incidence showed an increasing trend that was significant only in adults $\geq 50$ years (Figure 2B). Both $\mathrm{SgC}$ and $\mathrm{SgB}$ incidence decreased significantly in persons aged 0, 1-5, 6-14, and 15-24 years, but not in adults (Figure 2C and D). The decreasing trend in SgC IMD was similar in magnitude before and after the $\mathrm{SgC}$ vaccination recommendation in 2006 in all age groups. However, the decreasing trend for $\mathrm{SgC}$ was significantly steeper than for $\mathrm{SgB}$ in 1-5 year old children, the major vaccination target group $(p=0.001)$, and overall $(p=0.009)$, with no difference in other age groups (Figure $2 \mathrm{C}$ and $\mathrm{D}$ ).

When stratifying $\mathrm{SgC}$ incidence according to vaccination coverage, the decreasing trend for $\mathrm{SgC}$ in persons aged 1-24 years (age group targeted for vaccination including individual catch-up since 2006) was steeper in states with higher (Incidence rate ratio $(\mathrm{IRR})=0.83,95 \% \mathrm{Cl}: 0.80-0.86, p<0.0001)$ 
than lower vaccination coverage $(\mathrm{IRR}=0.89,95 \% \mathrm{Cl}$ : $0.86-0.93, p<0.0001 ; p$ for slope difference $=$ 0.01 ) (Figure 3). While this pattern was seen for all age groups within 1-24 years, differences in individual age groups were not statistically significant (online Table 2). In contrast, there were no differences in the decreasing trend for SgB IMD incidence according to vaccination coverage in 1-24 year olds (IRR $=0.92,95 \% \mathrm{Cl}: 0.89-0.95, p<0.0001$ and IRR $=0.93,95 \% \mathrm{Cl}: 0.90-0.96, p<0.0001$, respectively) or any other age group ( $p>0.23$ in all groups).

There was regional variation of IMD with on average higher total and SgB-incidence in Midwestern and Eastern states and highest SgC- and SgY-incidence in Midwestern and Southern states. These regional differences were statistically significant for $\mathrm{SgB}$ and $\mathrm{SgY}$, but not for $\mathrm{SgC}$ incidence (Table 3).

\section{Finetype diversity in relation to serogroup-specific IMD incidence and $\mathrm{SgC}$ vaccination}

Since recommendation of $\mathrm{SgC}$ vaccination, only a modest decrease occurred in the two most common $\mathrm{SgC}$ finetypes C:P1.5,2:F3-3 and C:P1.5-1,10-8:F3-6 (Figure 4). While the age distribution of cases with the most common finetype C:P1.5,2:F3-3 was similar to the overall $\mathrm{SgC}$ age distribution, C:P1.51,10-8:F3-6 occurred predominantly in young adults not targeted for vaccination, with only $25.0 \%$ of cases in children $<15$ years of age, compared to $51.1 \%$ overall. A disproportionate decrease was observed in the group of the 10 next most common $\mathrm{SgC}$ finetypes, which collectively made up a significant proportion of cases prior to introduction of $\mathrm{SgC}$ vaccination (Figure 4), from 49 cases in 2002 , to 32 in 2006, to 3 cases in 2010. For comparison, the number of cases with all other SgC finetypes decreased from 85 in 2002 to 83 in 2006 to 63 in 2010. This development was accompanied by a decrease in finetype diversity in SgC cases since 2006: From 2002 to 2005 the index of discrimination $D$ fluctuated between 0.82 and 0.86 , then decreased from 0.81 in 2006 to 0.69 in 2009, although increasing again to 0.76 in 2010 (Figure 5). The correlation between SgC incidence and finetype diversity from 2002 to 2010 was significant (Spearman's rho $=-0.88, p=0.002$ ). No such decrease in diversity was observed in $\mathrm{SgB}$ finetypes despite the decreasing trend in $\mathrm{SgB}$ incidence, with $D$ ranging from 0.93 to 0.97 during 2002-2010 (Figure 5), nor were incidence and diversity correlated (Spearman's rho $=-0.25, p=0.52$ ). The increase in $\mathrm{SgY}$ incidence in $>50$ year-olds after 2006 was not primarily due to an increase in the most common finetype Y:P1.5-2,10-1:F4-1 (10/11 finetyped SgY cases in 2005-2006 but 20/37 cases in 2007-2010), but rather to an increase in several rarer SgY finetypes, as was the isolated increase in 15-24 year olds in 2009 (Figure 2B). This was reflected in increased SgY-associated finetype diversity in 2007-2010 $(D=0.64-0.83)$ compared to 2003-2006 (0.48-0.57, Figure 5), but correlation between incidence and diversity was not statistically significant (Spearman's rho $=0.30, p=0.43$ ).

\section{Discussion}

Surveillance of IMD in Germany is nationwide and comprehensive, with a high proportion of cases undergoing serogrouping and genetic typing. Thus detailed epidemiological analyses required for public health decisions are possible. Available data suggest that the degree of underascertainment has decreased: A capture-recapture study that compared isolates analysed at NRLM and the statutory surveillance system estimated an underascertainment of $\sim 11 \%$ in the statutory system in $2003,{ }^{13}$ in keeping with the rather high proportion of NRLM cases that could not be matched to a notified case in 2002 and 2003. This proportion decreased markedly after direct reporting of NRL results to LHA (rather than only to primary laboratories) was instituted in 2004 and has remained low at $\sim 3 \%$ since 2006. However, an analysis of cases with either WHS or purpura fulminans notified to the LHA as suspected IMD, but not transmitted to the national level due to lack of laboratory confirmation, suggests additional underascertainment of $4-8 \%{ }^{14}$

The distribution of serogroups in our dataset was similar to that described in other European countries, ${ }^{1}$ and 15 with a preponderance of $\mathrm{SgB}$ cases, followed by $\mathrm{SgC}$ and $\mathrm{SgY}$ cases. According to national IMD surveillance data submitted to the European Centre for Disease Control and Prevention (ECDC), ${ }^{1}$ the number of reported SgB IMD cases in 24 European countries decreased by $\sim 33 \%$ from 2003 to 2009, comparable to observations in Germany (34\%). An overall 52\% decrease was observed in reported SgC IMD cases (51\% in Germany) in these countries. A marked decrease in the proportion of $\mathrm{SgC}$ IMD from $>30 \%$ to $<5 \%$ was described collectively for the 10 countries with $\mathrm{SgC}$ vaccination programmes. 
In an analysis of data from 6 European countries that implemented routine conjugate $\mathrm{SgC}$ vaccination, the reported decline in SgC IMD incidence in the years following implementation (Belgium (2002), Iceland (2002), Ireland (2000), Netherlands (2002), Spain (2000) and the UK (1999)) was more rapid than seen in Germany. ${ }^{16}$ However, baseline SgC IMD incidence prior to the vaccination recommendation was much higher than in Germany ( $1-4.5$ cases/100,000 inhabitants vs. $0.2-0.3$ cases $/ 100,000$ inhabitants), and all 6 performed catch-up vaccination campaigns in older children. ${ }^{16}$ Herd effects were later demonstrated in the UK and the Netherlands. ${ }^{17}$ and $18 \mathrm{SgC}$ incidence in these countries continues to be extremely low at 0.04 and 0.07 , respectively. ${ }^{1}$ Modelling studies in England suggest that carriage reduction achieved by the catch-up campaign, particularly in adolescents, who have the highest carriage rate ${ }^{19}$ and 20 remains instrumental in maintaining low IMD incidence in the UK ${ }^{21}$ This is supported by data reported to ECDC, ${ }^{1}$ according to which the proportion of SgC IMD among all serogrouped IMD cases in 2009 was $0 \%$ in Iceland, $0.5 \%$ in the UK, $5.1 \%$ in the Netherlands, $3.7 \%$ in Ireland, and $1.8 \%$ in Portugal, where catch-up campaigns targeted all persons up to 18 years or even older. In contrast, in Spain, where catch-up vaccination was limited to children 5 years and younger in 16/19 regions ${ }^{22}$ and Belgium, ${ }^{23}$ where catch up was limited to 1-6 year-olds in French regions and to 1-6 year olds and 15-18 year-olds in Flanders, the proportion of SgC IMD was higher at $7.1 \%$ and $13.8 \%$, respectively, but still lower than in Germany $(21.9 \%)$ or in countries that did not implement SgC vaccination before 2010 , e.g. France $(22.7 \%)$ or Denmark $(32.8 \%){ }^{1}$

The stronger decline in $\mathrm{SgC}$ than $\mathrm{SgB} I \mathrm{IMD}$ in children aged 1-5 years (i.e. the primary vaccination target group), the steeper decline of $\mathrm{SgC}$ cases in federal states with higher $\mathrm{SgC}$ vaccination coverage, and a significant decrease in the proportion of SgC IMD cases since 2006 suggest that vaccination is contributing to the decline in SgC IMD in Germany. However, interpretation is complicated by the already low and declining $\mathrm{SgC}$ incidence prior to implementation of routine $\mathrm{SgC}$ vaccination and the consequent low overall numbers of cases, limiting the power of our trend analyses before and after implementation of vaccination. Nonetheless, the impact of $\mathrm{SgC}$ vaccination appears limited compared to that seen in countries that implemented catch-up campaigns.

While primary data on vaccination coverage were only available for children at school entry from 2008 onwards for $13 / 16$ federal states, nationwide data on the number of prescribed vaccines was helpful in supplementing these data for the purpose of categorizing states according to higher or lower levels of $\mathrm{SgC}$ vaccine uptake. Unpublished results of a mail survey of 7078 German households participating in a representative health panel performed in 2009 by the market research company GfK

(http://www.gfk.de/) funded by Baxter and Novartis Vaccines showed regional variation similar to that observed with the data from school entry examinations and vaccine prescription data above. In this survey, vaccination coverage was highest in children aged $2-5$ years $(78.3 \%)$, followed by $6-10$ year olds (58.6\%), $0-1$ year olds (36.3\%), 11-16 year olds (36\%), and $17-18$ year olds (27.8\%)(F. Weidle, GfK, personal communication). These rates are much lower than those achieved in countries such as the UK ( $\geq 93 \%$ by second birthday since 2005 , and $\geq 85 \%$ among $5-17$ year old children in the schoolbased catch-up campaign ${ }^{21}$ ) or the Netherlands, in which coverage of $94 \%$ was achieved in 1-18 year old persons within the catch-up campaign. ${ }^{17}$ Thus, vaccination coverage attained through a recommendation for "individual"-based catch-up in Germany appears inadequate to achieve reduction of carriage in older children with the highest carriage rates, ${ }^{24}$ and thus to have a major impact on transmission. The relatively rapid loss of immunity after a single dose of $\mathrm{SgC}$ conjugate vaccine among children vaccinated at a young age $\mathrm{e}^{25}$ may further undermine population immunity in Germany.

The decrease in incidence of $\mathrm{SgC}$, but not $\mathrm{SgB} I M D$ was associated with a decrease in finetype diversity. The interplay between carriage and disease of meningococci is complex, ${ }^{26}$ involving carriage-induced immunity over time as well as virulence factors of carried strains. We hypothesize that strains causing IMD more frequently are also more frequently carried and/or transmitted in the population, thus requiring higher vaccination coverage to interrupt their transmission. In contrast, strains causing disease more rarely are presumably also associated with lower carriage/transmission rates, such that lower vaccination coverage might be sufficient to reduce transmission. This hypothesis is supported by the disproportionate decrease in rarer $\mathrm{SgC}$ finetypes observed after achievement of only moderate $\mathrm{SgC}$ vaccination coverage in Germany.

SgY IMD was uncommon, with the highest incidence in adolescents. However, the proportion of cases was highest in older adults, in whom incidence also increased significantly, albeit on a very low absolute level (Figure 2B). A recent increase in SgY incidence has been reported from several European countries, ${ }^{1 \text { and } 27}$ but this development does not seem to be a homogenous phenomenon. For instance, in Sweden, the increase was due mainly to an increase in finetype Y:P1.5-2,10-1:F4-1 in 
young adults, ${ }^{27}$ also the most common finetype in Germany. However, in Germany, the observed increase in SgY disease seen primarily in older adults was to a large extent due to cases with several different rarer $\mathrm{SgY}$ finetypes, as reflected in the increase in finetype diversity. In contrast, in England and Wales an increase in SgY IMD from 2007 to 2009 was associated primarily with finetype Y:P1.51,10-1:F4-1. The current epidemiological situation in Germany does not justify a general recommendation for SgY conjugate vaccination.

The high incidence of $\mathrm{SgB}$ disease in infants with a large proportion of cases in the first 6 months of life (59\% of infant cases) should be considered when developing vaccination strategies for newly developed $\mathrm{SgB}$ vaccines ${ }^{28}$ expected shortly on the European market. The overall low incidence of $\mathrm{SgB}$ IMD in Germany demands careful evaluation of costs and benefits in the development of $\mathrm{SgB}$ vaccination recommendations.

In conclusion, IMD incidence in Germany decreased to its lowest level in 2010 since initiation of mandatory surveillance. Our results suggest a very limited contribution of $\mathrm{SgC}$ vaccination to the decline in SgC IMD in Germany. The apparent stronger effect of only moderate vaccination coverage on IMD due to rarer versus more common SgC strains sheds light on the poorly understood transmission dynamics of IMD. Together with modelling studies based on effects seen in other countries with vaccination programmes, our results suggest that a more effective use of resources for $\mathrm{SgC}$ IMD vaccination could be achieved in Germany by specifically targeting adolescents in addition to 1 -year olds rather than recommending catch-up for all children up to age 17 on an individual basis.

\section{Funding}

No external funding was used for this research; however, the work of the National Reference Laboratory for Meningococci was funded by the Robert Koch-Institute.

A brief overview of the epidemiology of invasive meningococcal disease in Germany was presented as a Poster at the 11th Meeting of the European Meningococcal Disease Society in Ljubljana, Slovenia, 18-20 May, 2011.

\section{Conflicts of interest}

WH, OW and MD report no conflicts of interest.

JE received honoraria for lectures from Baxter $\mathrm{GmbH}$ and Novartis Vaccines and Diagnostics $\mathrm{GmbH}$.

MF participated in a scientific meeting sponsored by Novartis.

UV participated in Novartis and Glaxo Smith Kline advisory boards and scientific meetings sponsored by Novartis and Baxter. He received research support from Glaxo Smith Kline and Novartis for unrelated projects.

\section{Acknowledgements}

We thank Heike Claus for assistance with molecular typing.

\section{Appendix A. Supplementary data}

The following is the supplementary data related to this article: http://dx.doi.org/10.1016/j.jinf.2012.09.008. 


\section{References}

1. European Centre for Disease Prevention and Control. Surveillance of invasive bacterial diseases in Europe 2008/2009. Stockholm: ECDC; 2011.

2. Buysse CMP, Raat H, Hazelzet JA, Hulst JM, Cransberg K, Hop WCJ, et al. Long-term health status in childhood survivors of meningococcal septic shock. Arch Pediatr Adolesc Med 2008; 162(11):1036-41.

3. Robert Koch-Institute. Zur Situation bei ausgewählten Infektionkrankheiten in Deutschland: invasive Meningokokken-Erkrankungen im Jahr 2007. Epidemiol Bull 2008;32:265-72.

4. Sächsische Impfkommission. Sächsische Impfkommission beschließt Einführung der Varizellenund Meningokokkenimpfung C als Standardimpfung. Ärztebl Sachsen 2003;8(357):360.

5. Ständige Impfkommission am Robert Koch-Institut. Begründung der STIKO-Empfehlungen zur Impfung gegen Pneumokokken und Meningokokken vom Juli 2006. Epidemiol Bull 2006;31: 260-7. 6. Ständige Impfkommission am Robert Koch-Institut. Mitteilung der Ständigen Impfkommission am Robert Koch-institut: Empfehlungen der Ständigen Impfkommission (STIKO) am Robert Kochinstitut/stand: Juli 2006. Epidemiol Bull 2006;30:235-54.

7. Bundesministerium für Gesundheit. Gesetzliche Krankenversicherung Mitglieder, mitversicherte Angehörige und Krankenstand Jahresdurchschnitt 2010 [Statutory health insurance: members, coinsured family members and morbidity, 2010], http://www.bmg.bund.de/fileadmin/dateien/Downloads/ Statistiken/GKV/Mitglieder_Versicherte/KM1_Jahresdurchschnitt_2010.pdf; 2011 [cited 2011 Dec 5]. 8. Elias J, Health office in the rural district office wartburgkreis, Claus $\mathrm{H}$, Frosch M, Vogel U. Evidence for indirect nosocomial transmission of Neisseria meningitidis resulting in two cases of invasive meningococcal disease. J Clin Microbiol 2006; 44(11):4276-8.

9. Maiden MCJ, Bygraves JA, Feil E, Morelli G, Russell JE, Urwin R, et al. Multilocus sequence typing: a portable approach to the identification of clones within populations of pathogenic microorganisms. Proc Natl Acad Sci U S A 1998; 95(6):3140-5.

10. Hunter PR, Gaston MA. Numerical index of the discriminatory ability of typing systems: an application of Simpson's index of diversity. J Clin Microbiol 1988;26(11):2465-6.

11. Grundmann H, Hori S, Tanner G. Determining confidence intervals when measuring genetic diversity and the discriminatory Abilities of typing methods for microorganisms. J Clin Microbiol $2001 ; 39(11): 4190-2$.

12. Robert Koch-Institute. Impfquoten bei den Schuleingangsuntersuchungen in Deutschland 2009.

Epid Bull 2011;16:125-9.

13. Schrauder A, Claus H, Elias J, Vogel U, Haas W, Hellenbrand W. Capture-recapture analysis to estimate the incidence of invasive meningococcal disease in Germany, 2003. Epidemiol Infect 2006;135(4):1-8.

14. Robert Koch-Institute. Invasive Meningokokken-Erkrankungen: Retrospektive Erhebung von Verdachtsmeldungen nach x 6 ABs. 1 IfSG Deutschland, 2001e2007. Epid Bull 2009;(8):69-75. 15. Brehony C, Jolley KA, Maiden MCJ. Multilocus sequence typing for global surveillance of meningococcal disease. FEMS Microbiol Rev 2007;31(1):15-26.

16. Trotter CL, Ramsay ME. Vaccination against meningococcal disease in Europe: review and recommendations for the use of conjugate vaccines. FEMS Microbiol Rev 2007;31(1):101-7.

17. de Greeff SC, de Melker HE, Spanjaard L, Schouls LM, van DA. Protection from routine vaccination at the age of 14 months with meningococcal serogroup $C$ conjugate vaccine in the Netherlands. Pediatr Infect Dis J 2006;25(1):79-80.

18. Maiden M, Ibarz-Pavon A, Urwin R, Gray S, Andrews N, Clarke S, et al. Impact of meningococcal serogroup C conjugate vaccines on carriage and herd immunity. JID 2008;197(5):737-43.

19. Christensen H, May M, Bowen L, Hickman M, Trotter CL. Meningococcal carriage by age: a systematic review and meta-analysis. Lancet Infect Dis 2010;10(12):853-61.

20. Soriano-Gabarr_o M, Wolter J, Hogea C, Vyse A. Carriage of Neisseria meningitidis in Europe: a review of studies undertaken in the region. Expert Rev Anti Infect Ther 2011;9(9): 761-74.

21. Campbell H, Andrews N, Borrow R, Trotter C, Miller E. Updated postlicensure surveillance of the meningococcal $\mathrm{C}$ conjugate vaccine in England and Wales: effectiveness, validation of serological correlates of Protection, and modeling predictions of the duration of herd immunity. Clin Diagn Lab Immunol 2010;17(5):840-7.

22. Larrauri A, Cano R, Garcia M, de Mateo S. Impact and effectiveness of meningococcal C conjugate vaccine following its introduction in Spain. Vaccine 2005;23(32):4097-100.

23. Theeten $\mathrm{H}$, Van Damme $\mathrm{P}$, Carion F, Van Looveren $\mathrm{M}$. Vaccinatie tegen meningokokken $\mathrm{C}$ in België. Kroniek en context van een federaal-regionale campagne. Tijdschrift Voor Geneeskunde 2003;59(20):1239-51.

24. Claus H, Maiden MJC, Wilson DJ, McCarthy NDJ, Urwin R, Hessler F, et al. Genetic analysis of meningococci carried by children and young adults. J Infect Dis 2005;191:1263-71. 
25. Perrett KP, Winter AP, Kibwana E, Jin C, John TM, Yu LM, et al. Antibody persistence after serogroup $C$ meningococcalconjugate immunization of United Kingdom primary-school children in 1999_2000 and response to a booster: a phase 4 clinical trial. Clin Infect Dis 2010;50(12):1601-10. 26. Trotter CL, Gay NJ, Edmunds WJ. The natural history of meningococcal carriage and disease. Epidemiol Infect 2006;134(3): 556-66.

27. Thulin-Hedberg S, Törös B, Fredlund H, Olcén P, Mölling P.Genetic characterisation of the emerging invasive Neisseria meningitidis serogroup $Y$ in Sweden, 2000 to 2010. Euro Surveill 2011;16(23). Pii=19884.

28. Panatto D, Amicizia D, Lai PL, Gasparini R. Neisseria meningitides B vaccines. Expert Rev Vaccines 2011;10(9):1337-51. 


\section{Tables and Figures}

Table 1. Coverage with conjugate meningococcal $\mathrm{C}(\mathrm{SgC})$ vaccines at school entry 2009 , and prescribed number of conjugate SgC vaccine doses per 1000 children 2006-2010, Germany

\section{SgC vaccination coverage \\ Prescribed doses of SgC conjugate vaccine/1000 \\ at school entry one-year-old children ${ }^{\text {a }}$}

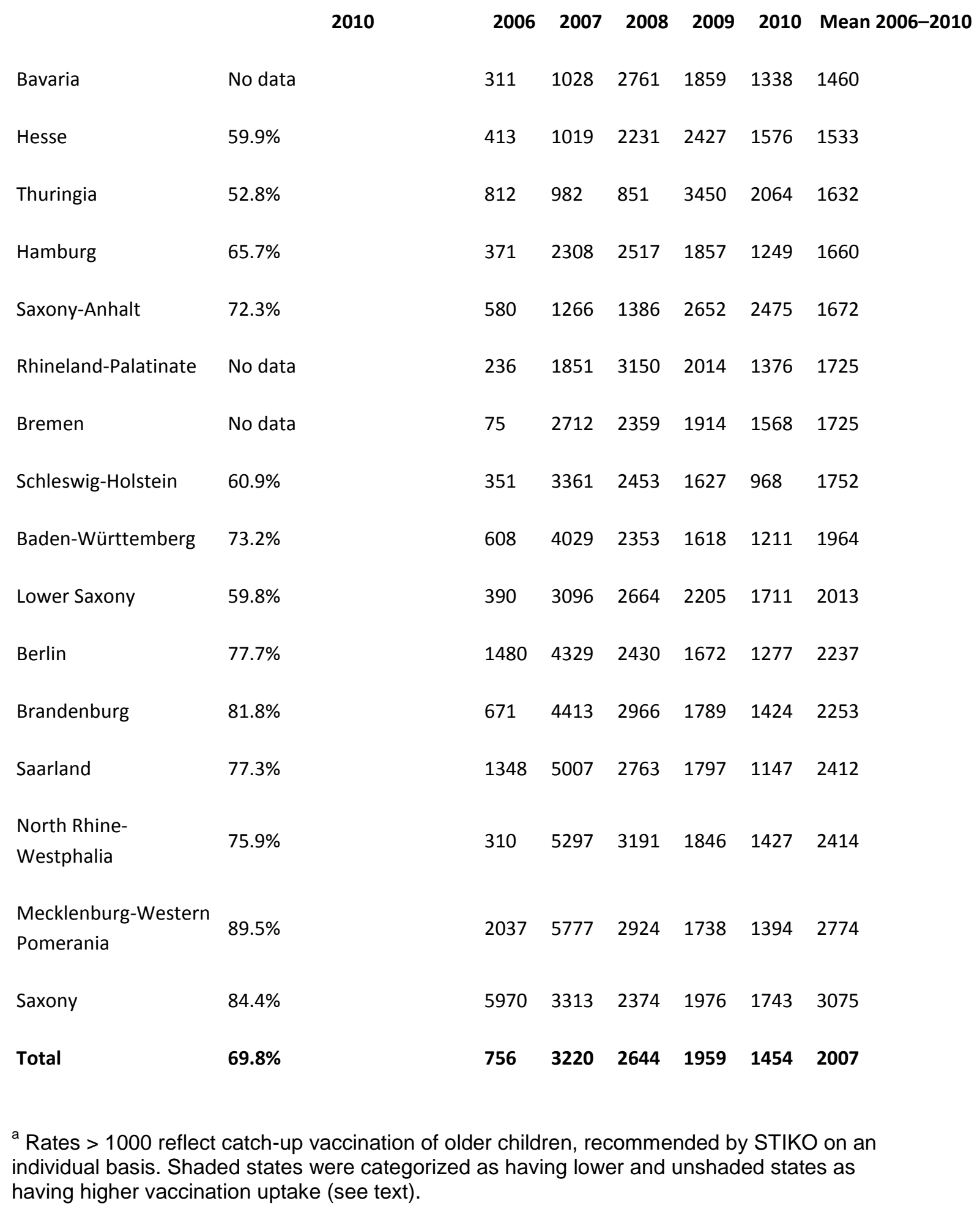


Table 2. Case fatality of invasive meningococcal disease according age and serogroup, Germany, 2002-2010 ( $n=441$ deaths).

\% Case fatality (95\% CI)

Age (years)

Serogroup C Serogroup B All serogroups

$0 \quad 9.9 \%(5.1-17.0 \%) \quad 9.1 \%(6.8-11.8 \%) \quad 9.3 \%(7.3-11.6 \%)$

$1-4 \quad 11.6 \%(7.7-16.5 \%) \quad 9.6 \%(7.5-12.0 \%) \quad 9.0 \%(7.4-10.9 \%)$

$5-14 \quad 5.7 \%(3.0-9.7 \%) \quad 3.8 \%(2.2-6.2 \%) \quad 4.2 \%(2.9-6.0 \%)$

$15-24 \quad 11.6 \%(8.3-15.5 \%) \quad 6.2 \%(4.7-8.1 \%) \quad 7.2 \%(5.9-8.7 \%)$

$25-49 \quad 15.2 \%(9.1-23.2 \%) \quad 8.6 \%(5.6-12.5 \%) \quad 10.1 \%(7.6-13.1 \%)$

$50+\quad 22.8 \%(15.7-31.2 \%) 15.9 \%(11.8-20.7 \%) 15.8 \%(13.0-19.1 \%)$

Total $\quad 11.9 \%(10.0-13.9 \%) 8.3 \%(7.3-9.3 \%) \quad 8.8 \%(8.0-9.6 \%)$

Table 3. Mean incidence of notified invasive meningococcal disease (cases/100,000 inhabitants) by serogroup and region, Germany, 2002-2010.

Northwest Midwest South East $p^{\underline{a}}$

$\begin{array}{llllll}\text { Serogroup C } & 0.164 & 0.179 & 0.183 & 0.155 & 0.09\end{array}$

$\begin{array}{lllllllll}\text { Serogroup B } & 0.456 & 0.519 & 0.369 & 0.613 & 0.0002\end{array}$

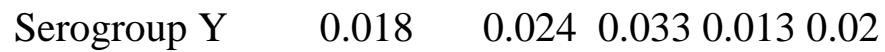

$\begin{array}{llllll}\text { Other Serogroups } 0.049 \quad 0.032 & 0.034 & 0.019 & 0.09\end{array}$

All serogroups $\quad 0.686 \quad 0.752 \quad 0.6180 .8000 .001$

Northwest: Lower Saxony, Bremen, Hamburg, Schleswig-Holstein; Midwest: Hesse, North RhineWestphalia, Rhineland-Palatinate, Saarland; South: Bavaria, Baden-Württemberg; East: Mecklenburg Western Pomerania, Brandenburg, Berlin, Saxony Anhalt, Saxony, Thuringia.

${ }^{a}$ Determined in negative binomial regression model.

Figure 1. Age distribution of notified IMD cases according to serogroup, Germany, 2002-2010. Numbers in bars denote the total number of cases notified from 2002 to 2010.

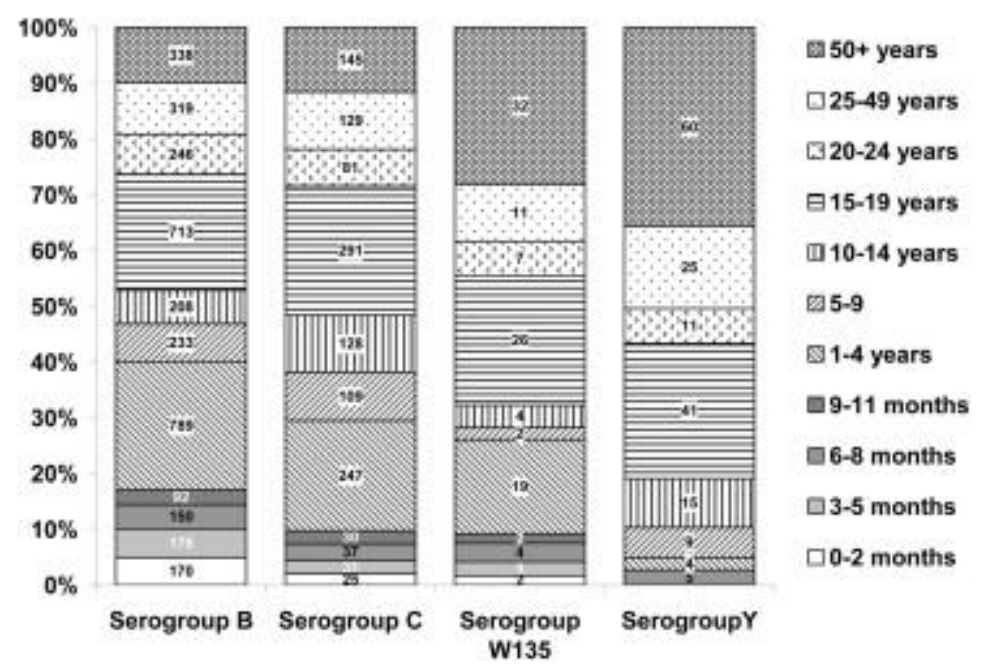


Figure 2.IMD incidence according to age and serogroup over time, 2002-2010. A: Total IMD incidence for $\mathrm{SgB}$ (Incidence rate ratio (IRR) $=0.93 ; 95 \% \mathrm{Cl}$ : $0.91-0.95, p<0.0001), \mathrm{SgC}(\mathrm{IRR}=0.89$; 95\%Cl: $0.86-0.91, p<0.0001)$ and SgY (IRR $=1.06 ; 95 \% \mathrm{Cl}: 1.00-1.12, p=0.05)$. B: Age-specific SgY incidence: IRR significant only in persons $\geq 50$ years (IRR $=1.18 ; 95 \% \mathrm{Cl}: 1.07-1.30, p=0.003$ ). C: Age-specific SgC incidence: Significant decrease in persons aged 0 (IRR $=0.90 ; 95 \% \mathrm{Cl}$ : $0.83-$ $0.97, p=0.007) 1-5$ (IRR $=0.81 ; 95 \% \mathrm{Cl}: 0.77-0.86, p<0.0001), 6-14(\mathrm{IRR}=0.84 ; 95 \% \mathrm{Cl}: 0.78-$ $0.90, p<0.0001$ ) and $15-24$ years (IRR: $0.91 ; 95 \% \mathrm{Cl}: 0.87-0.95, p<0.0001$ ), but not adults $\geq 25$ years (IRR $=1.00 ; 95 \mathrm{Cl}: 0.95-1.04, p=0.88)$. D: Age-specific SgB incidence: significant decrease in persons aged $0(0.94 ; 95 \% \mathrm{Cl}: 0.90-0.98, p=0.006), 1-5$ (IRR $=0.92 ; 95 \% \mathrm{Cl}: 0.88-0.95, p<0.0001)$, $6-14(\mathrm{IRR}=0.91 ; 95 \% \mathrm{Cl}: 0.86-0.96, p<0.0001), 15-24$ years $(\mathrm{IRR}=0.95 ; 95 \% \mathrm{Cl}: 0.92-0.97$, $p<0.0001$ ), but not in adults $\geq 25$ years (IRR $=0.98 ; 95 \% \mathrm{Cl}: 0.95-1.00, p=0.10$ ).
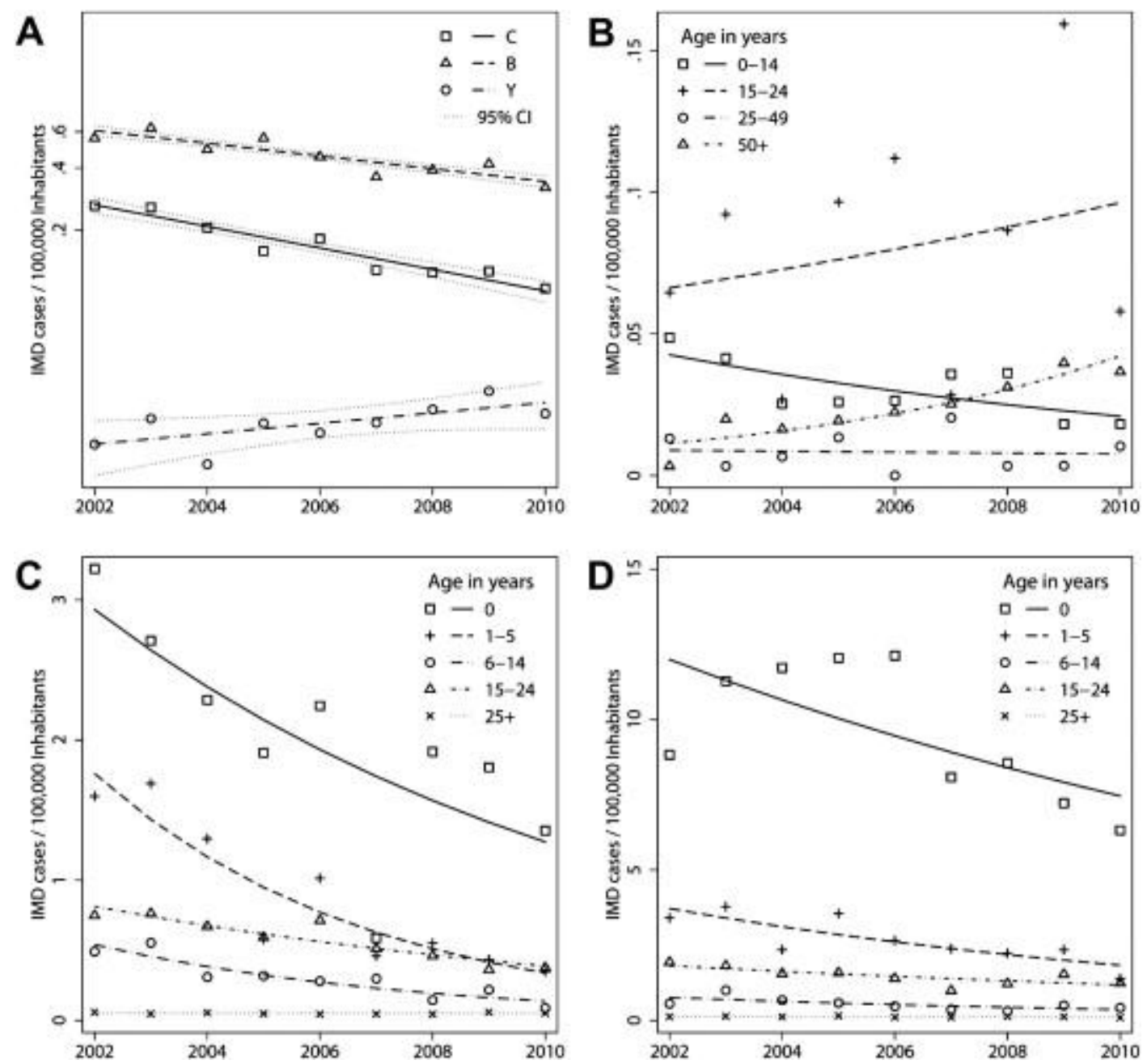
Figure 3. Incidence of IMD due to SgC in persons aged 1-24 years, 2002-2010, in states with lower (solid line) and higher (dotted line) vaccination coverage (Table 1) as modelled by negative binomial regression.

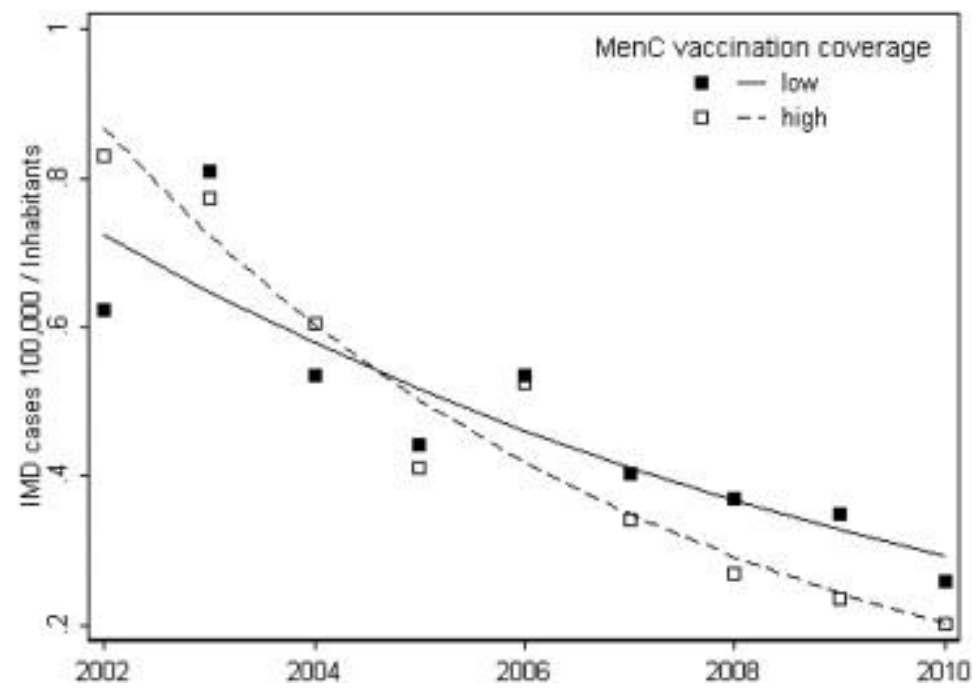

Figure 4. IMD cases due to serogroup C according to finetype by year, Germany, 2002-1010.

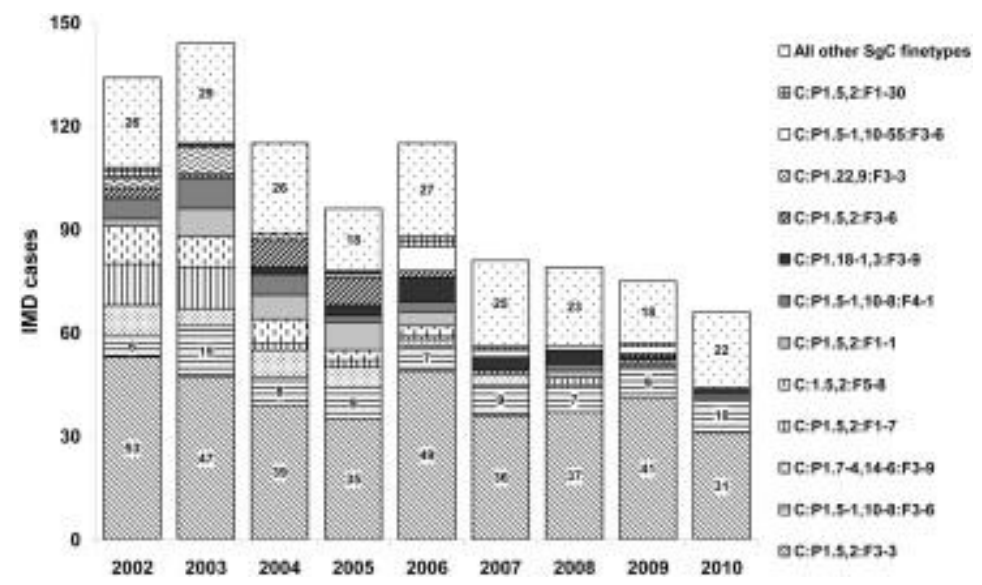

Figure 5. Index of discrimination for finetypes according to serogroups B, C and Y, Germany, 20022010. Note: Scale of $Y$ axes differs.
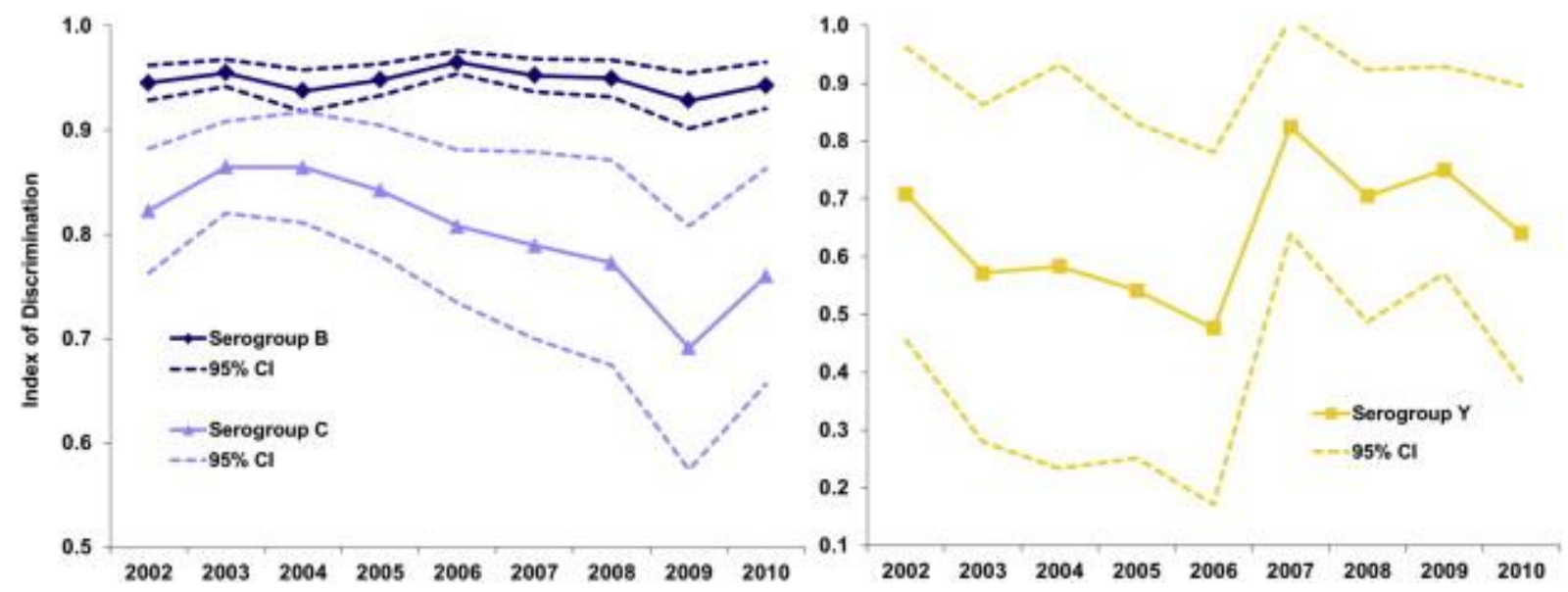